\title{
Research on Skin Texture Classification by Gray Level Co-occurrence Matrix and the BP Neural Network
}

\author{
Qiaohua Liu, Tianhua Chen, Xiaoyi Wang, Jiping Xu, Li Wang, Yinmao Dong and Hong Meng \\ No.11, Fucheng Road, Haidian District, Beijing, 100048, China
}

\begin{abstract}
It's very common to use the skin texture of gray level co-occurrence matrix to calculate the four most representative eigenvalues of human facial skin image: energy, moment of inertia, correlation and entropy. To test whether the four eigenvalues can represent the skin texture information, the article designed a verification experiment: the article used comparison data included arithmetic average roughness( $R a)$, average roughness(Rz), and smooth depth data(Rt) measured from DERMATOP V3 of CK in Germany, and experimental data included the four eigenvalues, to do principal component analysis, respectively, for unrelated principal component as the input data of BP neural network classifier. The experimental results show that using the four eigenvalues, the classification accuracy is higher. The method using gray level co-occurrence matrix to extract facial skin texture eigenvalue can relatively reflect the degree of human facial texture state than texture information measured by DERMATOP V3, which provides a simple and effective method for the data acquisition of skin texture.
\end{abstract}

Keywords- skin texture; gray level co-occurrence matrix; principal component analysis; BP neural network classifier

\section{INTRODUCTION}

Based on the theory of Traditional Chinese Medicine (TCM), the skin can reflect mental health status and other related information, which is widely recoglized both at home and abroad [1]. However, there are many factors affecting skin condition, each index value can reflect the different states of the skin. The texture of the skin is one of those fators, concretly emboding in facial wrinkles, such as forehead lines, crow's feet and lower eyelid wrinkles, vertical lines between the eyebrow and nasal root horizontal stripes, mouth week vertical lines, etc. Therefore, the research on skin texture has the vital significance to improve skin state.

At present, the study of skin texture mainly directly measures texture data (Ra, Rt, Rz), using ERMATOP V3 instrument from CK company. Paper [2] and [3] used the instrument to obtain texture data, and analyzed the correlation between them with age, facial position. The instrument is large, expensive and has complex operation, which caused great restrictions to research of skin texture research; In addition, because of intellectual property rights, the campany did not give data processing model, from the academic point of view, this method can neither refer, nor improve. In recent years, the analysis of the texture of image and feature extraction has been becoming a hot topic in the field of image processing, and has been proved to be a very good method theoretically. In paper [4] and [5], skin eigenvalues were extracted with gray level co-occurrence matrix (image data), and analyze the age, facial position's impact on these characteristic values. However, there is no study on the correlation of the two different source texture data, diversity, and their respective applicable scope. Therefore, this article designed a contrast experiment to analyze the characteristics from different source(image or instrument), in order to choose one that can accurately reflect the facial skin texture information.

In real life, the most obvious difference of the skin texture is embodied in gender. Generally speaking, male skin is coarseness, pore is bigger, texture is deeper; While women's is smooth, smaller and lighter. Theoretically, estrogen play an important role in skin wound repair, promoting hair growth, restraining the sebaceous glands secretion, delay skin aging, etc. In general, women than men excret much more estrogen than men [6]. So, no matter from the real life or in theory, the skin texture of men and women has bigger difference. Therefore, this article choose gender as a comparison standard, using characteristics data from the two different sources(image and instrument) as input of classifier, the higher classification accuracy of classifiers shows that this kind of data can reflect more information about skin texture based on gender features. Due to the characteristic value: energy, moment of inertia, relevance, entropy and Ra, Rz, Rt has very big correlation index, in order to eliminate redundant data from affecting the performance of classifier, the principal component analysis was carried out on the input data, respectively, for unrelated principal components as input of classifier.

\section{GRAY LEVEL CO-OCCURRENCE MATRIX AND EIGENVALUES EXTRACTION}

In this paper, the experimental data is 52 human facial images from China cosmetic research center of Beijing Technology and Business University, including 20 men's skin images, 32 women's. Camera system indicators: the lens magnification 50, with eight LED light source, resolution of $480 * 640$.

Gray level co-occurrence matrix reflects the comprehensive information about the direction, adjacent interval, and amplitude of variation [5], it is the basis of analyzing the skin texture and relevant characteristics. Assume that an image $f$, consists of $N_{x}$ pixels in the horizontal direction and $N_{y}$ vertical pixels, the most pixels in grayscale is $N_{g}$. Refer to: 


$$
\begin{aligned}
L_{x} & =\left\{1,2, \cdots, N_{x}\right\} \\
L_{y} & =\left\{1,2, \cdots, N_{y}\right\} \\
G & =\left\{1,2, \cdots, N_{g}\right\}
\end{aligned}
$$

The $\mathrm{G}$ is a transform of image $f$ from $L_{x} \times L_{y}$, every pixel in $L_{x} \times L_{y} \mathrm{~h}$ as a corresponding gray value, refer to: $L_{x} \times L_{y} \rightarrow G$.

Assumed that:

$$
\begin{array}{r}
P_{x}(i)=\sum_{j=1}^{N} P(i, j) ; i=1,2, \cdots, N \\
P_{y}(j)=\sum_{i=1}^{N} P(i, j) ; j=1,2, \cdots, N
\end{array}
$$

where $i, j$ are the image intensity values of the image. $P(i, j)$ are the grayscale value of spatial positions $(i, j)$. Eigenvalues are extracted from the co-occurrence matrix $P(i), P(j) .14$ kinds eigenvalues were proposed by the paper [7], and current research and experimrntal results show that the energgy, moment of inertia, relevance, the entropy are the four that can be fully characterized skin texture information.

\section{1) Energy}

Energy is also called angular second moment (ASM), is a kind of image gray uniformity measure, namely:

$$
Q_{1}=\sum_{i}^{N} \sum_{j}^{N}[P(i, j)]^{2}
$$

ASM is the sum of squares of gray level co-occurrence matrix element values, it is one of the important indicators to measure gray distribution uniformity, is also seen as measure of the image texture thickness.

\section{2) Entropy(ENT)}

Entropy is a kind of measure image with the amount of information. Texture is kind of image information, if a digital image texture has no texture, and gray level co-occurrence matrix matrix is almost zero.

$$
Q_{4}=-\sum_{i} \sum_{j} P(i, j) \log [P(i, j)]
$$

Entropy reflects the complexity of the image texture, and the distribution of randomness and the amount of information.

\section{3) Inertia moment}

Inertia moment , also known as contrast, can be understood as the sharpness of the image. The contrast measures gray scale variation of local image. It's value reflects the image clarity, density and degree of grooving depth texture.

$$
Q_{2}=\sum_{k=1} k^{2}\left[\sum_{i} \sum_{j} P(i, j)\right]
$$

where, $k=i-j$.

\section{1) Correlation (COR)}

Correlation is used to measure the similarity degree of the gray level co-occurrence matrix of row or column elements.

$$
Q_{3}=\frac{\sum_{i} \sum_{j} i j P(i, j)-\mu_{x} \mu_{y}}{\sigma_{x} \sigma_{y}}
$$

where:

$$
\begin{aligned}
& \left\{\begin{array}{c}
\mu_{x}=\sum_{i} i \sum_{j} P(i, j) \\
\mu_{y}=\sum_{j} j \sum_{i} P(i, j)
\end{array}\right. \\
& \left\{\begin{array}{l}
\sigma_{x}{ }^{2}=\sum_{i}\left(i-\mu_{x}\right)^{2} \sum_{i} \sum_{j} P(i, j) \\
\sigma_{y}{ }^{2}=\sum_{j}\left(j-\mu_{y}\right)^{2} \sum_{i} \sum_{j} P(i, j)
\end{array}\right.
\end{aligned}
$$

where $\mu_{x}, \sigma_{x}$ is respectively the mean and variance of $P_{x}(i, j) . \mu_{y}, \sigma_{y}$ is the mean and variance of $P_{y}(i, j)$.

Correlation measures the local texture image grayscale linear correlation, reflects the similarity between the texture region in a certain direction.

In this paper, energy, entropy, moment of inertia and the correlation are extracted from the gray level co-occurrence matrix, respectively in four directions, $0^{\circ}, 45^{\circ}, 90^{\circ}, 135^{\circ}$, and the average value of the four are shown in table 1 in the following section.

\section{PRINCIPAl COMPONENT ANALYSis (PCA)}

The amount of eigenvalues has very important influence on the classification accuracy of classifiers. The best way to maintain or improve the performance of the neural network classifier is to choose the minimun number of eigenvalue subset[8]. The four eigenvalue this paper selected and the arithmetic average roughness $\mathrm{Ra}$, average depth of the roughness $\mathrm{Rz}$, smooth $\mathrm{Rt}$ measured by $\mathrm{CK}$ instrument, has significant correlation. 
Principal component analysis (PCA) can effictively reduce the dimension of raw data, in addition to retaine the most information of the original data. PCA can overcome the defects of traditional dimension reduction method that simply delete some of original data [9]. This paper uses principal component analysis (pca) to extract the classifier inputs.

TABLE I. TEXTURE DATA FROM DIFFERENT SOURCES

\begin{tabular}{lccccc}
\hline \multicolumn{1}{c}{ No. } & $\mathbf{1 0 0 1}$ & $\mathbf{1 0 0 2}$ & $\ldots$ & $\mathbf{2 0 3 1}$ & $\mathbf{2 0 3 2}$ \\
\hline Energy & 0.31630 & 0.22927 & $\ldots$ & 0.22836 & 1.46062 \\
\hline Entropy & 1.48743 & 1.94431 & $\ldots$ & 2.05381 & 0.22737 \\
\hline $\begin{array}{l}\text { inertia } \\
\text { moment }\end{array}$ & 0.18859 & 0.34274 & $\ldots$ & 0.47326 & 1.88834 \\
\hline Correlation & 1.94625 & 1.09776 & $\ldots$ & 0.89652 & 0.36255 \\
\hline Ra $(\mathrm{mm})$ & 0.02232 & 0.02095 & $\ldots$ & 0.02125 & 0.02490 \\
\hline $\mathrm{Rz}(\mathrm{mm})$ & 0.08539 & 0.08578 & $\ldots$ & 0.08270 & 0.10075 \\
\hline $\mathrm{Rt}(\mathrm{mm})$ & 0.17492 & 0.16167 & $\ldots$ & 0.13860 & 0.19991 \\
\hline
\end{tabular}

Because those eigenvalues have different units, the eigenvalue has to be standardized before PCA. And Ra, Rz, Rt parameters have been standardized, in the range of $[0,1]$, as Table 2:

TABLE II. PCA ANALYSIS OF TOTAL VARIANCE

\begin{tabular}{c|c|c|c|c}
\hline \multirow{2}{*}{ Data } & $\begin{array}{c}\text { Componen } \\
\mathbf{t}\end{array}$ & Total & $\begin{array}{c}\text { Variance } \\
\mathbf{\%}\end{array}$ & $\begin{array}{c}\text { Accumulative } \\
\mathbf{\%}\end{array}$ \\
\hline \multirow{2}{*}{$\begin{array}{c}\text { Data } \\
\text { extracted } \\
\text { from } \\
\text { image }\end{array}$} & 1 & 2.276 & 56.904 & 56.904 \\
\cline { 2 - 5 } & 2 & 1.675 & 41.872 & 98.776 \\
\cline { 2 - 5 } & 3 & 0.04 & 1.006 & 99.782 \\
\hline \multirow{2}{*}{$\begin{array}{c}\text { Data from } \\
\text { CK } \\
\text { instrument }\end{array}$} & 1 & 0.009 & 0.218 & 100 \\
\cline { 2 - 5 } & 2 & 0.046 & 1.527 & 97.908 \\
\hline
\end{tabular}

If the variance of component is greater than 1 , then the component will be choosed as the main component. The Table 2 shows that the first two of image data are the main component, their variance are respectively 2.276, 1.675, and the first one of the instrument data is the main component, its variance is 2.937 .

The second and third column of Table 3 are not the eigenvector of the principal component, that is to say , they are not the coefficient of the main component 1 and main component 2. Coefficient of principal component is each independent component load vector divided by independent component characteristic value of characteristic vector arithmetic square root. According to texture eigenvalue, the principal component 1 (set as $y_{1}$ ) and principal component 2 (set as $y_{2}$ ), the function equation is:

$$
\begin{gathered}
y_{1}=0.659 \times x_{1}-0.535 \times x_{2}+0.526 \times x_{3}-0.053 \times x_{4} \\
y_{2}=-0.064 \times x_{1}+0.449 \times x_{2}+0.460 \times x_{3}-0.764 \times x_{4}
\end{gathered}
$$

where $x_{1}, x_{2}, x_{3}, x_{4}$ are the standardized value of ASM, ENT, Interia moment and COR.
Set the principal component of Ra, Rz, Rt as $y$,

$$
y=0.575 \times R_{a}+0.580 \times R_{z}+0.576 \times R_{t}
$$

\begin{tabular}{|c|c|c|c|c|}
\hline \multicolumn{5}{|c|}{ Data extracted from image } \\
\hline & \multicolumn{2}{|c|}{ Component } & \multicolumn{2}{|c|}{ eigenvector } \\
\hline & 1 & 2 & 1 & 2 \\
\hline ASM & 0.437 & -0.049 & 0.659 & -0.064 \\
\hline ENT & -0.355 & 0.347 & -0.535 & 0.449 \\
\hline Inertia & 0.349 & 0.355 & 0.526 & 0.46 \\
\hline COR & -0.035 & -0.59 & -0.053 & -0.764 \\
\hline \multicolumn{5}{|c|}{ Data from CK instrument } \\
\hline & & Component & \multicolumn{2}{|c|}{ eigenvector } \\
\hline & & 1 & \multicolumn{2}{|c|}{1} \\
\hline Ra & & 0.986 & \multicolumn{2}{|c|}{0.575} \\
\hline $\mathrm{Rz}$ & & -0.994 & \multicolumn{2}{|c|}{0.58} \\
\hline Rt & & -0.988 & \multicolumn{2}{|c|}{0.576} \\
\hline
\end{tabular}

where $R_{a}, R_{z}, R_{t}$ are the standardized value of $\mathrm{Ra}, \mathrm{Rz}, \mathrm{Rt}$.

TABLE III. COMPONENT MATRIXA

IV. The Design AND Training of The SKIn TeXture OF CLASSIFIER

BP neural network classification equipment can realize the function of the complex nonlinear mapping, self-study, fault tolerant and generalization ability [9]. So, this article choosed the $\mathrm{BP}$ artificial neural network classifier.

The amount of the input nodes of BP network classifier is the number of input vector dimensions, the output is the classification results. Hidden layer nodes can determined by the experience formula of $\mathrm{l}=2 \mathrm{n}-1$ (where $\mathrm{N}$ is the input vector dimension), learning rate and the number of training sets to 0.01 and 1000 . Training data, 52 group of samples, is from the Chinese cosmetics research center of Beijing besiness and technology university, using the average as the final classification accuracy comparison, such as Table 4:

\section{TABLE IV. CLASSIFICATION ACCURACY OF BP NETWORK}

\begin{tabular}{lll}
\hline $\begin{array}{l}\text { classification } \\
\text { accuracy }\end{array}$ & Image data & Instrument data \\
\hline 1 & 0.7973 & 0.6553 \\
\hline 2 & 0.7667 & 0.6145 \\
\hline 3 & 0.8 & 0.5667 \\
\hline 4 & 0.7773 & 0.6216 \\
\hline 5 & 0.8145 & 0.5853 \\
\hline Avarage & 0.79116 & 0.60868 \\
\hline
\end{tabular}

\section{V.EXPERIMENTAL RESULTS AND ANALYSIS}

The experimental results shows that the four eigenvalue extracted from gray level co-occurrence matrix of image as input data of the BP neural network classifier, the classification accuracy is higher. Therefore, using gray level co-occurrence matrix to extract human facial skin image texture eigenvalue 
can better reflect the degree of facial texture state, than directly using the instrument measured texture data on gender characteristics. This study provides a more simple and more effective method to acquisit the skin texture of data. To some extent, it provides convenience and conditions for the in-depth research on skin texture, at the same time, provides a new way to rich skin texture data.

According to the texture image, this paper selected gender as the characteristics to do the comparative experiments, follow-up study can compare the image data and instrument data in age, facial parts (forehead, eyes, cheeks, chin) characteristics of performance, to find out the correlation, differences and scope of application between the image data and instrument data, and provide theoretical support for skin care and other follow-up study.

\section{ACKNOWLEDGEMENT}

This work was financially supported by Comprehensive Reform Project of Talent Cultivation of Beijing Technology and Business University_ Research Center Construction of Cosmetics Synergy in China (No.19005428069/007). The Importation and Key projects of science and technology plan of Beijing Municipal Institutions (KZ201510011011). Those supports are gratefully acknowledged.

\section{REFERENCES}

[1] Meng Hong, Yinmao Dong, Congfen He. How to apply theory of Chinese medicine to the cosmetics technology [C] / / The beauty of traditional Chinese medicine branch of China association of Chinese medicine academic conference. 2009.

[2] Li Tang. Women's skin texture, roughness, elastic correlation with age and areas of research [D]. Sichuan university, 2006.

[3] Xiang Wen, Xian Jiang, Caiyun Bian etc. Three methods of noninvasive evaluation of the relationship between female age and skin texture, roughness, elasticity [J]. Journal of sichuan university, medical sciences, 2009, 40 (2) : 364-366.

[4] Wang Sujing, Chun-guang Zhou, Na Zhang, etc. Based on the shape and texture characteristics of human face age estimation [J]. Journal of Jilin university: engineering science, 2011, 41 (5) : 1383-1387.

[5] Sheng Han. Research on the relationship between the lateral forearm flexor skin texture parameters and age [D]. Chongqing medical university, 2012

[6] Chun Wu, Yanlong Lee, Fan Zhang. The research progress of Chinese medicine skin aging resistance [J]. Chinese journal of traditional Chinese medicine, 2014, (9) : 2889-2891.

[7] Schmidt S, Koch B, Schulz R, et al. Comparative analysis of the applicability of the skeletal age determination methods of Greuli-ch-Pyle and Thiemann-Nitz for forensic age estimation in livings ects [J]. International journal of legal medicine, 2007, 121(4): 293-296.

[8] Pengyi Gao. The BP neural network classifier optimization technology research [D]. Huazhong university of science and technology, 2012.

[9] Qin Huang, Wei Chang, Yiliang Liu etc. The BP neural network classifier based on PCA [J]. Journal of chongqing institute of technology: natural science edition, 2009, 23 (7) : 89-96.

[10] FuQing Bu. Image recognition and classification based on artificial neural network [D]. Chengdu technology university, 2010. 